\title{
HST OBSERVATIONS OF JETS AND RADIO LOBES
}

\author{
PHILIPPE CRANE \\ European Southern Observatory \\ Karl Schwarzschildstraße 2 \\ D85748 Garching \\ Germany
}

The Hubble Space Telescope has proved to be remarkably useful for discovering and for studying the optical counterparts of radio lobes and radio jets. Since much of the structure seen in the radio is found on subarcsecond scales, it is not surprising that HST, with it's improved resolution relative to ground based observations, would be a major contributor to this field of research. This paper reports briefly on some of these successes, and refers to more detailed descriptions in the literature.

\section{Pictor A Hot Spot}

The western hot spot of Pictor A was already known to be highly polarized from the ground based observations(see ref. [1]). Figure 1. shows the polarization map of the western hot spot of Pictor A obtained with HST Faint Object Camera utilizing the F342W filter and the 3 polarizers[2]. The peak count in the F96 images was 13 counts obtained in 900s! Nevertheless, since the FOC is basically a noiseless detector, good quality polarization maps were obtained. Figure 1 shows that the magnetic field vectors are nearly perpendicular to the jet axis. The peak polarization exceeds $60 \%$ and indicates that the magnetic field structure in the hot spot has been resolved on a scale of $70 \mathrm{pc}$.

\section{The Jet in $3 \mathrm{C264}$}

This jet was discovered by HST[3]. Here we present an overlay of a recently obtained MERLIN map and the HST image. There appears to be a slight misalignment of the radio jet axis from the optical jet axis. A VLA map at $2 \mathrm{~cm}$ and MERLIN maps at $6 \mathrm{~cm}$ and at $18 \mathrm{~cm}$ have been obtained. The spectral index map between the $2 \mathrm{~cm}$ and and $18 \mathrm{~cm}$ maps appears to confirm the misalignment above. The details of these observations will be reported in a forthcoming publication[4]. 


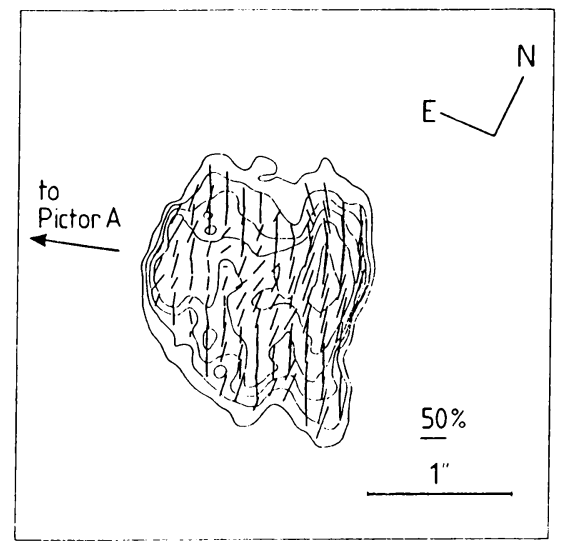

Figure 1. Polarization map of the western hot spot of Pictor A. The resolution is about $70 \mathrm{pc}$. The lines show the orientation of the magnetic field.

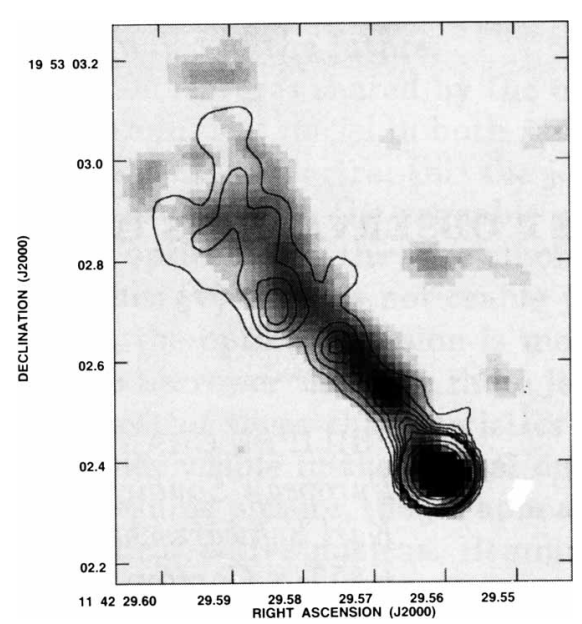

Figure 2. Overlay of HST image and MERLIN $6 \mathrm{~cm}$ map(contours).

\section{NGC6251-Confirmation of a UV feature}

This source which has one of the longest and best studied radio jets[5] has been observed at two different epochs with the preCostar FOC F96 camera and 3 different filters. The second epoch observations confirm the previous report [6] of a UV feature $\operatorname{close}(\approx 0.2 \operatorname{arcsec})$ to the nucleus of NGC6251 and at a position angle of roughly $90^{\circ}$ to the axis of the radio jet. The UV feature does not appear in the $\mathrm{V}$ or $\mathrm{B}$ band images but only in the UV F $342 \mathrm{~W}$ images. The origin of this feature remains to be determined. It's discovery is a testament to the value of UV high resolution imaging.

\section{PKS 0812+02}

This fascinating quasar radio source[7] at a redshift $z=0.402$ has been observed with WFPC2 with a filter that avoids the main emission line features. The images reveal resolved structure coincident with the end of the radio jet. This may be similar to the radio lobe in $3 \mathrm{C} 33[8]$. Further observations are planned which include both continuum and emission lines.

\section{References}

[1] Röser H.J., and Meisenheimer, K., (1987) Ap. J.314,70.

[2] Thomson, R.C., Crane,P., and Mackay,C.D., (1995) Ap.J. Letters,446,L93.

[3] Crane, P., Peletier, R., et al., (1993) Ap.J. Letters,402,L37.

[4] Crane,P., Pedlar, A., et al.,(1996) in preparation.

[5] Perley,R., Bridle, A.H., and Willis, A.G.,(1984) Ap. J. Suppl.,54,291.

[6] Crane,P., 1993 in Jets in Extragalactic Radio Sources, edited by H.J. Röser and K. Meisenheimer, (Berlin: Spinger)pp 223.

[7] Wyckoff, S., et al.(1983) Ap. J.265,43.

[8] Crane,P., and Stiavelli,M.,(1992) MNRAS257,17P. 